\title{
ON THE STRUCTURE OF THE ENVELOPE OF NOVA DELPHINI 1967 IN THE NEBULAR STAGE
}

\author{
ROMUALD TYLENDA
}

Astronomical Institute, Nicolaus Copernicus University, Toruń, Poland

\begin{abstract}
The analysis of line profiles in the spectrum of Nova Del 1967 in the nebular stage shows that the polar axis of the envelope is longer than the equatorial one. The polar caps are probably cooler and thinner than the equatorial rings.
\end{abstract}

\section{Introduction}

The aim of this paper is to show how variations of physical conditions between particular regions of the Nova Del 1967 envelope in the nebular stage can explain the observed profiles of emission lines, and to estimate the magnitude of these variations. The following lines are treated: $\mathrm{H} \beta$ (or $\mathrm{H} \gamma$ ), [O III] $\lambda \lambda 4363,4959,5007 \AA$, [Ne III] $\lambda 3869 \AA$ and $[\mathrm{Ne} \mathrm{v}] \lambda 3426 \AA$.

Observations were made by Dr A. Woszczyk at McDonald Observatory from May to August, 1969. The spectra were taken at dispersions of $17 \AA \mathrm{mm}^{-1}$ and $3 \AA \mathrm{mm}^{-1}$. The detailed description of the observational material and the preliminary interpretation will be published elsewhere (Tylenda and Woszczyk, 1975).

Each unblended line in the spectrum of Nova Del consists of six components, which have been marked with numbers from 1 to 6 towards longer wavelengths in Figure 1. Relative intensities of particular maxima are different for different lines.

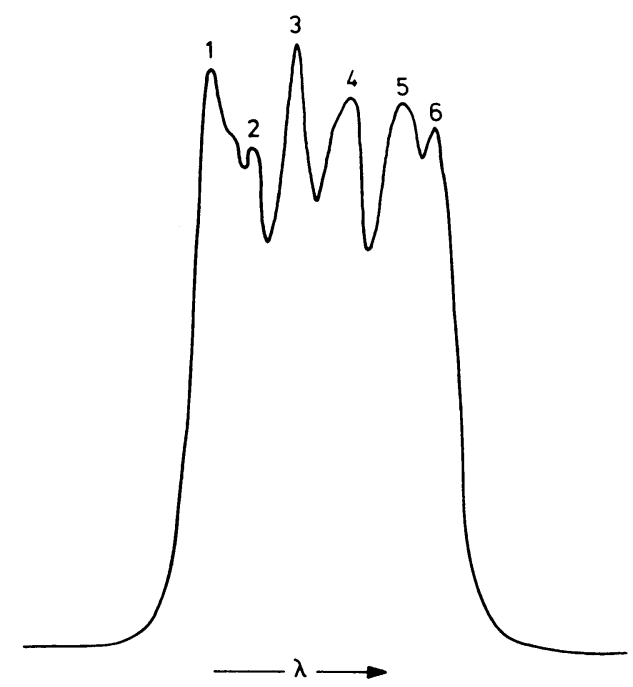

Fig. 1. Line profile for $\mathrm{H} \beta$ emission line. 
Roughly speaking, the higher the ionization potential of a particular element, the smaller are the intensities of maxima 3 and 4 relative to the remaining ones. In this paper, the measurements of intensities of components $2,3,4,5$, and 6 are relative to the intensity of the first one for the above-mentioned lines.

It is assumed that each maximum is produced by one condensation in the nova envelope and that these regions are homogeneous; physical conditions are constant in each condensation.

\section{Evaluation of Formulae}

Let us consider an $i$ th region of the nova envelope at a distance $R_{i}$ from the central star (Figure 2) and let $\theta_{i}$ be the angle between the line of sight and the radius of the region. A thickness $\Delta R$ of the envelope is assumed to be constant throughout the envelope. After simple considerations, one obtains

$$
I_{i} \Delta \lambda_{i} \cong j_{i} V_{i},
$$

where $I_{i}$ is the intensity of the spectral line from the $i$ th region, $j_{i}$ is the emissivity per unit volume, and $V_{i}$ is the volume of the $i$ th region. Because of the high expansion

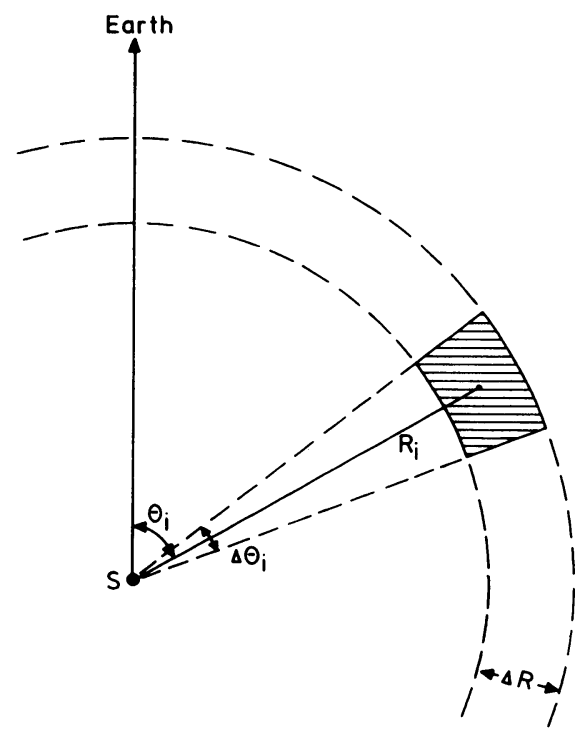

Fig. 2. Evaluation of formulae in Section 2.

velocity ( $\sim 580 \mathrm{~km} \mathrm{~s}^{-1}$ ) of the envelope, $V_{i}$ is a volume of a region contained between two cones with angles $\theta_{i}-\Delta \theta_{i}$ and $\theta_{i}+\Delta \theta_{i}$. Thus

$$
V_{i}=2 \pi R_{i} \sin \theta_{i} R_{i} \Delta \theta_{i} \Delta R .
$$

It is assumed that the whole envelope was ejected in one moment. This is consistent with Malakpur's (1974) recent investigations. 
After simple evaluations, one obtains the relation

$$
\frac{I_{i}}{I_{1}}=\frac{j_{i} R_{i}}{j_{1} R_{1}}
$$

Let us assume an electron temperature $T_{\mathrm{e}}$ and an electron density $N_{\mathrm{e}}$ in the $i$ th region. The emissivity from the unit of volume in a Balmer line arising from $m$ th excitation level is equal to

$$
j_{m}=\text { const } N_{\mathrm{e}}^{2} \frac{b_{m}\left(T_{\mathrm{e}}\right)}{T_{\mathrm{e}}^{3 / 2}} \exp \left(\frac{h v_{m}}{k T_{\mathrm{e}}}\right) \text {. }
$$

Using relations (3) and (4), one obtains

$$
n_{i}^{2}=\frac{H_{m, i} t_{i}^{3 / 2}}{\varrho_{i}} \frac{\exp \left(\frac{h v_{m}}{k T^{1}}\right)}{\exp \left(\frac{h v_{m}}{k t_{i} T^{1}}\right)} \frac{b_{m}\left(T^{1}\right)}{b_{m}\left(t_{i} T^{1}\right)}
$$

where

$$
\begin{aligned}
n_{i} & \equiv \frac{N_{\mathrm{e}, i}}{N_{e, 1},} \\
t_{i} & \equiv \frac{T_{\mathrm{e}, i}}{T_{\mathrm{e}, 1}}, \\
\varrho_{i} & \equiv \frac{R_{i}}{R_{1}}, \\
T^{1} & \equiv T_{\mathrm{e}, 1}, \quad N^{1} \equiv N_{\mathrm{e}, 1}, \\
H_{m, i} & \equiv \frac{I_{m, i}}{I_{m, 1}}
\end{aligned}
$$

( $I_{m, i}$ is the intensity of the $i$ th maximum of a Balmer line arising from the $m$ th excitation level.)

The ratio of the sum of intensities of the lines of [O III] $\lambda \lambda 4959$ and $5007 \AA$, to the intensity of [O III] $\lambda 4363 \AA$ is a function of the electron temperature and density:

$$
\frac{I_{5007}+I_{4959}}{I_{4363}}=E\left(T_{\mathrm{e}}, N_{\mathrm{e}}\right)=\mathrm{const} \frac{f_{2}\left(T_{\mathrm{e}}, N_{\mathrm{e}}\right)}{f_{3}\left(T_{\mathrm{e}}, N_{\mathrm{e}}\right)} .
$$

Let us introduce an observational value

$$
R_{i} \equiv\left(\frac{I_{5007}+I_{4959}}{I_{4363}}\right)_{i} /\left(\frac{I_{5007}+I_{4959}}{I_{4363}}\right)_{1}=\frac{2.93 r_{5007, i}+r_{4959, i}}{3.93 r_{4363, i}},
$$

where $r_{\lambda, i} \equiv I_{\lambda, i} / I_{\lambda, 1}$. In the last expression the theoretical value of the ratio $I_{5007} /$ $I_{4959}=2.93$ is used (observations of Nova Del give the value 2.8-3.0). 
Using (8) one obtains

$$
R_{i}=\frac{E\left(t_{i} T^{1}, n_{i} N^{1}\right)}{E\left(T^{1}, N^{1}\right)}
$$

The emissivity in the line $\left[N_{\mathrm{e}} \mathrm{III}\right] \lambda 3869 \AA$ may be expressed by the following formula:

$$
j_{3869}=\text { const } N_{\mathrm{Ne} \text { III }} \frac{f_{2, \mathrm{Ne} \text { II }}\left(T_{\mathrm{e}}, N_{\mathrm{e}}\right)}{f_{\mathrm{Ne} \text { III }}\left(T_{\mathrm{e}}, N_{\mathrm{e}}\right)} \text {. }
$$

A similar expression may be written for the line [Ne v] $\lambda 3426 \AA$. We now introduce the observed value

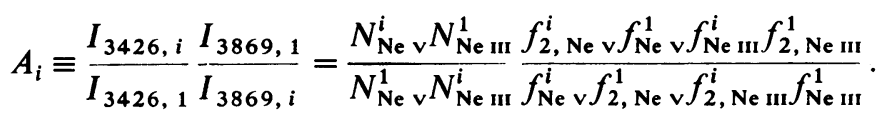

The equation of ionization equilibrium for $\mathrm{Ne}$ III in the $i$ th region of the envelope has the form

$$
4 \pi N_{\mathrm{Ne} \text { III }}^{i} \int_{v_{1}}^{\infty} \frac{L_{v}}{16 \pi^{2} R_{i}^{2}} \exp \left(-\tau_{v, i}\right) a_{v} \mathrm{~d} v=N_{\mathrm{e}}^{i} N_{\mathrm{Ne} \text { IV }}^{i} \alpha_{\mathrm{Ne} \text { III }}^{i} .
$$

One can write a similar equation for the ionization of Ne IV. Assuming $\tau_{v, i}$ to be the same for all regions and neglecting a dependence of the recombination coefficient $\alpha$ on the electron temperature, we get the relation

$$
\frac{N_{\mathrm{Ne} \mathrm{v}}^{i} N_{\mathrm{Ne} \mathrm{III}}^{1}}{N_{\mathrm{NeV}}^{1} N_{\mathrm{Ne} \mathrm{III}}^{i}}=\frac{1}{\varrho_{i}^{4} n_{i}^{2}},
$$

which together with (12) gives the formula

$$
\varrho_{i}^{4}=\frac{1}{A_{i} n_{i}^{2}} \frac{f_{2, \mathrm{Nev}}^{i} f_{\mathrm{Ne} \mathrm{v}}^{1} f_{\mathrm{Ne} \mathrm{m}}^{i} f_{2, \mathrm{Ne} \mathrm{III}}^{1}}{f_{\mathrm{Nev}}^{i} f_{2, \mathrm{Nev}}^{1} f_{2, \mathrm{Ne} \mathrm{m}}^{i} f_{\mathrm{Ne} \mathrm{II}}^{1}}
$$

\section{Results}

The observational values are presented in Table $\mathrm{I} . H_{i}$ quantities have been evaluated from $\mathrm{H} \beta$ and $H \gamma$. It should be mentioned that, in the line [Ne v] $\lambda 3426 \AA$, maxima 2 and 6 have been blended by maxima 1 and 5 respectively. The values $A_{2}$ and $A_{6}$ have been determined assuming $I_{3426,2}=I_{3426,1}$ and $I_{3426,6}=I_{3426,5}$. Therefore, the results obtained for regions 2 and 6 should be taken as less certain than those for the other ones.

Observational data have been put into expressions (5), (10), and (15) and values for $n_{i}, t_{i}$, and $\varrho_{i}$ have been computed. Seaton's (1959) values of $b_{m}\left(T_{\mathrm{e}}\right)$ have been used. Functions $f, f_{2}$, and $f_{3}$ occurring in (8) and (15) were evaluated by Gorbatzky and 
TABLE I

Observational data

\begin{tabular}{lll}
$i$ & $H_{i}(\mathrm{H} \beta)$ & $H_{i}(\mathrm{H} \gamma)$ \\
\hline 1 & 1.00 & 1.00 \\
2 & $0.82 \pm 0.1$ & $0.86 \pm .02$ \\
3 & $1.12 \pm .01$ & $1.07 \pm .01$ \\
4 & $0.96 \pm .01$ & $0.97 \pm .01$ \\
5 & $0.96 \pm .02$ & $1.00 \pm .02$ \\
6 & $0.89 \pm .02$ & $0.95 \pm .02$ \\
\hline$i$ & & \\
\hline & $R_{i}$ & $A_{i}$ \\
\hline & 1.00 & 1.00 \\
3 & $0.99 \pm .05$ & $(1.12 \pm .03)$ \\
4 & $1.30 \pm .04$ & $0.66 \pm .02$ \\
5 & $1.26 \pm .05$ & $0.70 \pm .03$ \\
6 & $0.92 \pm .05$ & $1.13 \pm .06$ \\
& $0.86 \pm .04$ & $(1.26 \pm .09)$
\end{tabular}

Minin (1963). Transition probabilities and collision strengths for forbidden lines have been taken from Garstang (1968) and Czyzak et al. (1968), respectively. In all above expressions $N^{1}$ and $T^{1}$ are free parameters. Computations have been performed using $N^{1}=4.5 \times 10^{6} \mathrm{~cm}^{-3}$ and $T^{1}=1.15 \times 10^{4} \mathrm{~K}$ (Malakpur, 1973). The values of these parameters are not important because the results are almost independent of variations of $N 1$ and $T 1$.

Table II contains the results of the computations together with their errors resulting from observational errors in $H_{i}, R_{i}$ and $A_{i}$ (Table I). The agreement of results

TABLE II

Results of computations $\left(\mathrm{N}^{1}=4.5 \times 10^{6} \mathrm{~cm}^{-3}, T^{1}=1.15 \times 10^{4} \mathrm{~K}\right)$

\begin{tabular}{|c|c|c|c|}
\hline$i$ & $n_{i}$ & $t_{i}$ & $Q_{i}$ \\
\hline \multicolumn{4}{|c|}{$H_{i}$ derived from $\mathrm{H} \beta$} \\
\hline 1 & 1.00 & 1.00 & 1.00 \\
\hline 2 & $0.91 \pm .01$ & $1.04 \pm .02$ & $1.02 \pm .01$ \\
\hline 3 & $0.97 \pm .01$ & $0.92 \pm .02$ & $1.12 \pm .01$ \\
\hline 4 & $0.89 \pm .01$ & $0.95 \pm .02$ & $1.15 \pm .02$ \\
\hline 5 & $1.01 \pm .02$ & $1.03 \pm .03$ & $0.97 \pm .02$ \\
\hline 6 & $0.99 \pm .02$ & $1.07 \pm .03$ & $0.96 \pm .03$ \\
\hline \multicolumn{4}{|c|}{$H_{i}$ derived from $\mathbf{H} \gamma$} \\
\hline 1 & 1.00 & 1.00 & 1.00 \\
\hline 2 & $0.93 \pm .01$ & $1.03 \pm .02$ & $1.01 \pm .01$ \\
\hline 3 & $0.94 \pm .01$ & $0.92 \pm .01$ & $1.13 \pm .02$ \\
\hline 4 & $0.90 \pm .01$ & $0.95 \pm .02$ & $1.14 \pm .02$ \\
\hline 5 & $1.03 \pm .01$ & $1.02 \pm .03$ & $0.96 \pm .02$ \\
\hline 6 & $1.03 \pm .01$ & $1.06 \pm .03$ & $0.94 \pm .03$ \\
\hline
\end{tabular}




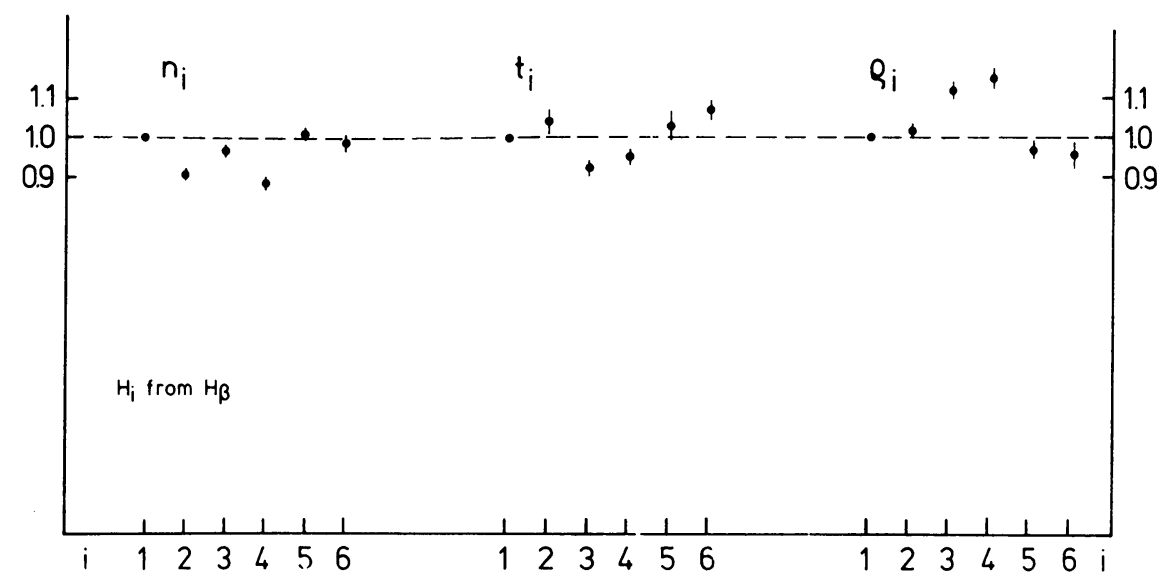

Fig. 3. Results of computations; see Table II.

obtained using $\mathrm{H} \beta$ and $\mathrm{H} \gamma$ observations is very good. Some of the values from Table II are presented in Figure 3.

The above results show that maxima 3 and 4 are produced by regions of the nova envelope which are about $7 \%$ thinner, $7 \%$ cooler and $13 \%$ more distant from the central star than the others. Adopting Malakpur's (1973) geometrical model of the Nova Del envelope, we conclude that the polar axis of the envelope is longer than the equatorial one. This is consistent with results obtained for other novae by Mustel and Boyarchuk (1970).

In future the author intends to extend the investigations by including the influence of the geometry of the nova envelope on line profiles, and a helium ionization structure which determines the optical thickness of the envelope in a wavelength range in which $\mathrm{Ne}$ III and $\mathrm{Ne}$ IV are ionized.

\section{References}

Czyzak, S. J., Krueger, T. K., Martins, P. de A. P., Saraph, H. E., Seaton, M. J., and Shemming, J.: 1968, in D. E. Osterbrock and C. R. O'Dell (eds.), 'Planetary Nebulae', IAU Symp. 34, 138.

Garstang, R. H.: 1968, in D. E. Osterbrock and C. R. O'Dell (eds.), 'Planetary Nebulae', IAU Symp. 34, 143.

Gorbatzky, V. G. and Minin, I. N.: 1963, Nestatzionarnyie Zvezdy (Unstable Stars), Moscow, p. 73. Malakpur, I.: 1973, Astron. Astrophys. 24, 125.

Malakpur, I.: 1974, Astrophys. Space Sci. 27, 467.

Mustel, E. R. and Boyarchuk, A. A.: 1970, Astrophys. Space Sci. 6, 183.

Seaton, M. J.: 1959, Monthly Notices Roy. Astron. Soc. 119, 90 .

Tylenda, R. and Woszczyk, A.: 1975, Bull. Astron. Obs. Torun' (in press). 